\title{
El cuento: estrategia didáctica para la comprensión
}

\section{lectora}

The story: didactic strategy for reading comprehension

Aracelly Fernanda Núñez-Naranjo. ${ }^{1}$, Alexandra Wilma Paredes Guevara. ${ }^{2}$, Verónica Pérez.

${ }^{3} \&$ Karina Raquel Yancha Yancha. ${ }^{4}$

\begin{abstract}
.
DOI: https://doi.org/10.33262/concienciadigital.v4i1.2.1583

Introduction. This study was based on an analysis carried out on elementary basic general education students of the Mario Cobo Barona Educational Unit, due to the fact that they had difficulties when reading and understanding, a situation that was associated with low academic performance, low motivational classes and traditionalists limiting the student's creativity and intellectual growth. Objective. Strengthen the capacity for reflection, analysis, comprehension, argumentation and synthesis of the texts read. Methodology. The work had a mixed, empirical, descriptive and analytical approach, which allowed by means of the application of instruments to identify the main causes of this problem. Results. As the main results, it was obtained that $60 \%$ of the students did not consider reading as an alternative for improvement and enjoyment, $25 \%$ sometimes do so with comic books or stories. $82 \%$ of the

\footnotetext{
1 Universidad Tecnológica Indoamérica, Carrera de Educación Básica, Ambato, Ecuador. alexandraparedes@uti.edu.ec, https://orcid.org/0000-0001-7431-2339.

2 Universidad Tecnológica Indoamérica, Carrera de Educación Básica, Ambato, Ecuador. fernandanunez@indoamerica.edu.ec, https://orcid.org/0000-0002-0185-5516.

3 Universidad Tecnológica Indoamérica, Carrera de Educación Básica, Ambato, Ecuador. veronicaperez@indoamerica.edu.ec, https://orcid.org/0000-0002-0476-4193.

4 Universidad Tecnológica Indoamérica, Ambato, Ecuador. karinayancha@gmail.com
} 
students mentioned that the classes are not motivated by interesting readings according to their age, and $75 \%$ indicated that after the short readings the teachers ask questions about the text, but that in long readings they prefer to send essays or writing, in this way history was established as a didactic strategy framed in motivation, reading comprehension and material resources that in a simple, practical way and according to the age of the student. Conclusion. It was concluded that reading comprehension is fundamental in the reality of the classroom, in all areas of knowledge and in the different school stages and that the motivation to read involves a series of efforts that can be executed through didactic strategies such as the story.

Keywords: Reading comprehension, Didactics, Didactic strategies, History.

\section{Resumen.}

Introducción. Este estudio se basó en un análisis realizado a los estudiantes de educación general básica elemental de la Unidad Educativa Mario Cobo Barona, debido a que presentaban dificultades al momento de leer y comprender, situación que se vio asociada un bajo rendimiento académico, clases poco motivacionales y tradicionalistas limitando en el estudiante la creatividad y su crecimiento intelectual. Objetivo. Fortalecer habilidades de reflexión, análisis, comprensión, argumentación y síntesis de los textos leídos. Metodología. El trabajo tuvo un enfoque mixto, empírico, descriptivo y analítico, lo que permitió por medio de la aplicación de instrumentos identificar las principales causas de esta problemática. Resultados. Cómo principales resultados se obtuvieron que al $60 \%$ de los estudiantes no consideraba a la lectura como una alternativa de superación y gusto, el $25 \%$ a veces lo hace con revistas cómicas o cuentos cortos. El 82\% de los estudiantes mencionó que las clases no son motivadas con lecturas de interés acorde a su edad, y el 75\% indicó que después de las lecturas cortas los docentes realizan preguntas sobre el texto, pero que en lecturas largas prefieren enviar ensayos o redacciones, de esta manera se estableció como estrategia didáctica el cuento enmarcadas en la motivación, comprensión lectora y recursos materiales que, de forma sencilla, práctica y que acorde a la edad del educando. Conclusión. Se concluyó que la comprensión lectora es fundamental en la realidad del aula, en todas las áreas de conocimiento y en las diferentes etapas escolares y que la motivación a la lectura conlleva una serie de esfuerzos que pueden ejecutarse por medio de estrategias didácticas como el cuento.

Palabras claves: Comprensión lectora, Didáctica, Estrategias didácticas, El cuento

\section{Introducción.}

Yubero y Larrañaga (2015) mencionan que los estudiantes no tienen hábitos lectores, ni leen de forma voluntaria afectando de forma directa a las necesidades del estudiante en afrontar el mundo que es imprescindible y repercute en su desenvolvimiento a futuro. Tirado, Santos y Tejero-Díez (2013), menciona que son muy pocos los docentes que dentro de su aula de 
clase motivan a los estudiantes en la lectura y compresión de textos, debido a que esto implica introducir cambios para fomentar la lectura y crear un papel en el cual la lectura se convierta en una transformación para la educación.

Ecuador, es un país en pro de mejorar la calidad de vida educativa de sus educandos, para ello ha creado normativas y procedimientos que buscan la calidad de la educación (Acurio Ponce \& Nuñez Naranjo, 2019), motivar a la lectura es una propuesta innovadora, pues intervienen distintos factores que permiten el desarrollo del pensamiento. La Constitución de la República del Ecuador menciona que son parte del patrimonio cultural tangible e intangible las lenguas, formas de expresión, tradición oral y diversas manifestaciones, así como los documentos, objetos, colecciones, archivos, bibliotecas, las creaciones artísticas, científicas y tecnológicas (Asamblea Nacional Constituyente del Ecuador, 2008), por ello importante el desarrollo habilidades desde las poblaciones más jóvenes en: reflexión, análisis, comprensión, argumentación y síntesis, así la educación es un instrumento de transformación de la sociedad desde el núcleo familiar y desde la escolarización, ya que contribuye al desarrollo de los habitantes por medio de proceso de aprendizaje fortalecidos (Asamblea Nacional de la República del Ecuador, 2010).

La Unidad Educativa Mario Cobo Barona ubicada en la provincia de Tungurahua Ecuador, ha presentado un bajo rendimiento académico de sus educandos en áreas como lenguaje y comunicación y desarrollo del pensamiento, esta situación radica en las pocas estrategias metodológicas aplicadas con los estudiantes en el aula de clase, actividades tradicionales poco motivadoras. En un análisis preliminar, las principales dificultades asociadas a su bajo rendimiento fueron la dificultad que perciben los profesores de sus educandos al momento de leer, analizar, e interpretar los textos literarios, escasa comprensión, análisis, síntesis y argumentación.

Ramírez Leyva (2009) menciona que leer y lectura, pueden entenderse como una forma simplificada y compleja pues comprende actos de decodificación y comprensión asociadas a representaciones gráficas, mientas que Hoyos-Flórez y Gallego (2017) menciona que la lectura debe ser interactiva, activa y motivante para captar el atención del lector y que este pueda ser asociado a conocimientos previos permitiéndole inferir, interpretar y entender lo que lee. Es así que la lectura y el pensamiento crítico son habilidades indispensables para comprender un mundo globalizado (Flores Guerrero, 2016).

Alonso y Mateos (1985) establece que los factores que interviene en la lectura, se podrían resumir en: motivación para la lectura, desarrollo de la lengua materna y madurez apropiada, la primera basada en el interés por lo que se lee, la segunda en el conocimiento adecuado y amplio del lenguaje con el que se comunica y, por último, una madurez evolutiva adecuada para lo que se lee y por lo que se lee, además propone los modelos: ascendente, también llamado bottom up; modelo en el que la persona se inicia por las letras y los conjuntos de estas, hasta conseguir entender las unidades más amplias como las palabras y el texto 
completo, se centra en el texto y se basa en la decodificación; descendente, también llamado top down; modelo que busca las palabras o frases globales, para después, realizar un análisis de los elementos que lo componen, este modelo que tuvo el acierto de apuntar a que no solo existe el texto y su decodificación; sino también, las experiencias previas de las personas que leen y, el Interactivo, en el que se integran los dos anteriores, donde la lectura es significativa para las personas; es decir, que las personas sepan evaluar su propio rendimiento, a través de los objetivos que guían la lectura, lo que implica la presencia de un lector activo que procese el texto (Ferreri, 2015).

Aprender a leer no supone un proceso mecanizado de enseñanza, sino el aprendizaje fundamental con múltiples estrategias que facilitan la relación de la información contenida en el texto y los conocimientos de quien lee, para edificar la representación del significado global del contexto (Mendoza Velazco, 2018).

Jouini \& Saud (2005) establecen que la comprensión lectora es la habilidad de leer y comprender textos literarios, es así que la naturaleza constructivista de la lectura enlaza el proceso de leer con la construcción de significados esto permite que el lector, se encuentre activo por tanto el producto será la comprensión del texto leído, por tanto "la interacción entre el lector y el texto es el fundamento de la comprensión” (Redondo Gonzalez, 2008).

Marina, (2014) menciona que la educación en el constructivismo ha ocupado un papel importante, pues referencia a la fundamentación epistemológica y a las múltiples teorías psicológicas que tienen en común planteamientos y a los desarrollos educativos en el aula de clase, a los docentes o tutores quienes las emplean y a las estrategias didácticas y establece que Vygotsky mencionaba que "el instrumento para internalizar o hacer su propia cultura, la historia y la sociedad era el lenguaje", mientas que para Kistemaker y Broeder (2014) el lenguaje es un organizador de la conducta, la cual se modifica en la medida en que los seres humanos escriben y leen, y se desarrollan habilidades de un pensamiento superior, pues pueden razonar, analizar, sintetizar y concluir, empleando el lenguaje que posee, por tanto según Xhemajli (2016)el rol de un docente es la de ser un tutor en los intercambios y dar sentido a las intervenciones, ser facilitador y moldeadores del aprendizaje de los estudiantes y para ello tiene a su medio diferentes estrategias didácticas.

Para Thomson (2012), la palabra estrategias es muy común en el lenguaje, las estrategias no son fáciles de crearlas ya que se requiere de habilidades y destrezas. La estrategia es un método de planificación que, al aplicarlo a un conjunto de acciones, permite alcanzar un objetivo que sirve para verificar varios resultados.

Acurio Ponce y Núñez-Naranjo (2019), mencionan que las estrategias didácticas son un conjunto de actividades planificadas para el proceso de enseñanza y aprendizaje enfocadas a comunicar ideas y a proyectar aprendizajes en momentos pedagógicos basados en técnicas, procedimientos, con el uso de recursos que tienen como finalidad fortalecer los aprendizajes. 
Una estrategia didáctica es un proceso organizado y orientado que permite obtener y alcanzar los objetivos, la aplicación de esta permite que en la vida diaria mejoren los procedimientos y técnicas diseñados para el proceso enseñanza y aprendizaje.

Existen estrategias que según Bermúdez, Casanova y Villareal (2015) y Ospina Rodríguez, (2006) permiten implementar motivar en la ejecución de una clase, como: escuchar a los estudiantes de manera activa, apelar a las vivencias y emociones, explicar en qué van a consistir las actividades que realizarán, despertar la curiosidad, presentar material didáctico, permitir la interacción entre estudiantes y docente, realizar preguntas y responderlas, de manera que puedan aclarar ideas inconclusas, incentivar el trabajo en equipo, señalar lo positivo de sus actividades, no poner énfasis en sus fracasos y facilitar la percepción de que se actúa con autonomía. Anaya-Durand \& Anaya-Huertas (2010), mencionan que dentro de la motivación académica se deben revisar tres componentes: el contexto de la clase, los sentimientos y creencias de los estudiantes y los comportamientos, pues esto permite al docente, identificar el nivel motivaciones de sus estudiantes en clase ya que esto interviene en las acciones positivas o negativas al momento de realizar las actividades académicas.

Para motivar a la lectura, según Carrasco Altamirano (2003) y Vega et al. (2014), se pueden aplicar estrategias antes de lectura como: muestreo, en el que el lector imagina una parte del texto, ideas o imágenes e interpreta su contenido; predicción por medio del conocimiento en el que el lector tiene del mundo le facilita pronosticar que pasara al final de un cuento o la continuación de una carta, por lo tanto, la predicción es la capacidad para suponer lo que va a ocurrir en la lectura de ¿cómo será un texto?, ¿cómo continuará o cómo puede terminar, utilizando pistas gramaticales, lógicas o culturales?. Se puede señalar que, en la lectura, se necesita estar activo y se puede adelantar a lo que dice el texto, las predicciones se realizan por imágenes, títulos, subtítulos, marcas, colores, información que el docente les comparte a sus estudiantes, se realiza preguntas sobre los personajes del cuento, entre otras, es fundamental como se planteen las preguntas para poder iniciar la lectura. Esta estrategia también se usa durante la lectura, con la confirmación, autocorrección y los adelantos del lector, mientras que las estrategias durante la lectura, permiten deducir el contenido de la lectura, plantear preguntas sobre el texto, formular predicciones, verificarlas y rechazarlas, aclarar posibles dudas o significados de palabras y las estrategias después de la lectura se realizan por medio de actividades que enfocan la comprensión, la reconstrucción y análisis de los significados del texto.

Parra Delgado (2008), menciona que el cuento posee como regla general la imaginación y fantasía, con una narración clara, fluida, sencilla comprensión y sirven para relajar al niño, Serrabona (2008) indica que el cuento permite la maduración psicológica y sociológica del niño y establece que es un relato breve, con hechos imaginarios y reales que despierta la imaginación y curiosidad. 
El cuento facilita el aprendizaje armónico, para Jiménez Ortíz y Gordo Contreras (2014), existen diferentes géneros de cuentos entre ellos: fantasía, terror, miedo, poder, así mismo estos géneros ayudan en la formación de los niños y niñas, el cuento de ser didáctico pasa a ser poético, imaginativamente rico, narra personajes, actitudes, aptitudes, sentimientos, valores, entre otros (Padial Ruz \& Sáenz-López, 2013).

Para Vigotsky el lenguaje llega a convertirse en un instrumento de comunicación que fortalece la conciencia personal y social (Marínez Chepe, Tocto, \& Palacios, 2015). Por lo general un cuento se compone por tres grupos: los personajes, los lugares y los objetos, lo que facilita un proceso de enseñanza y aprendizaje por medio de la comunicación (Marínez Chepe, Tocto, \& Palacios, 2015).

El cuento constituye una estrategia para el fortalecimiento de la comprensión lectora en los niños y niñas, por ser activo y constructivo en la interpretación del significado del texto del cuento de esta manera se influye en la adquisición del proceso lector (Rojas Camargo \& Prieto Martínez, 2017); es activo porque se deben de poner en juego una serie de operaciones y estrategias mentales para procesar la información que se recibe del cuento, y es constructivo porque las operaciones y estrategias puestas en juego permiten construir el significado de lo que quiere decir el cuento creando así una nueva información de la interacción entre la información obtenida del texto y el conocimiento previo del lector, de esta manera el cuento, permite fantasear, crear personajes, jugar con la imaginación, construir mundos mejorando la expresión, formas de comunicación, reforzando el habla, el aprendizaje colaborativo y la comprensión lectora (Jiménez Ortíz \& Gordo Contreras, 2014).

\section{Metodologia.}

El trabajo tuvo un enfoque mixto, empírico, descriptivo y analítico, lo que permitió por medio de la aplicación de instrumentos identificar las principales causas de esta problemática. La población de estudio correspondió a 5 docentes y 40 estudiantes de la Unidad Educativa Mario Cobo Barona, para la obtención de la información se aplicaron fichas de observación y encuestas estructuradas.

\section{Resultados.}

Cómo principales resultados se obtuvieron que al $60 \%$ de los estudiantes no considera a la lectura como una alternativa de superación y gusto, el 25\% a veces lo hace con revistas cómicas o cuentos cortos. El 82\% de los estudiantes indicó que en las clases no son motivados con lecturas de interés acorde a su edad, además el 75\% indicó que después de las lecturas cortas los docentes realizan preguntas sobre el texto, pero que en lecturas largas prefieren enviar ensayos o redacciones. Un 45\% de los estudiantes mencionó que los textos largos como libros no contienen imágenes por lo que las lecturas largas son cansadas. El 70\% de los estudiantes indicó algunos profesores a veces realizan actividades de lectura que son interesantes y el 55\% de los estudiantes indicó que participa activamente cuando el profesor 
realiza preguntas sobre el texto leído. Los instrumentos aplicados a los estudiantes muestran resultados desalentadores ya que con en su mayoría los estudiantes no muestran interés por la lectura llevando a eso bajas en la compresión lectora asociado a la aplicación de estrategias didácticas poco motivacionales por partes del profesorado. Los instrumentos aplicados aprecian una realidad existente impuesto por un sistema escolarizado.

\section{Conclusiones.}

- Frente al mundo actual en el que se desenvuelven los educando se han ido creando hábitos de ocio entre ellos en internet y la televisión esto ha generado una actitud negativa frente a la lectura, es por ello que se debe establecer estrategias o actividades destinadas a ser trabajadas en la comprensión de textos y más en los pequeños con la lectura de cuentos (Prieto, 2021). Fomentar en los estudiantes la capacidad de análisis, entendiéndose como la facultad de comprender a partir de deferencias y desagregar ordenadamente sus partes, estableciendo relaciones entre las mismas, y como segunda la capacidad de síntesis, el saber unir diferentes elementos en un todo significativo, de tal modo que bajo el análisis y síntesis acompañado de la lectura constante se convierte en una parte fundamental para desarrollarse a futuro (Benavides, 2013).

- La comprensión lectora es fundamental en la realidad del aula, en todas las áreas de conocimiento y en las diferentes etapas escolares, por tanto, comprender cualquier texto según el nivel de desarrollo del individuo implica el nivel de comprensión literal, inferencial y crítico, aplicando varias estrategias de manera hacer relación de sus conocimientos previos y los nuevos (Aquino, 2020). Sin embargo, la falta de gusto por leer por parte de los estudiantes puede derivarse de factores como: bajos niveles educativos por parte de los padres, bajos recursos económicos, material de lectura llamativo o interesante acorde a la edad. Hay estudiantes que viven en contextos diferentes por lo que resulta difícil acceder a libros digitales (Carranza, 2018).

- Motivar a la lectura conlleva una serie de esfuerzos que pueden ejecutarse por medio de estrategias didácticas que permitan la comprensión lectora, de esta manera cuento como estrategia didáctica resulta ser útil para trabajar en diferentes áreas del conocimiento, siempre y cuando haya la pertinencia entre el contenido y la parte de fantasía, considerando la realidad en la que viven nuestros educandos en el ámbito escolar, la importancia del papel que toman los estudiantes al ser agentes de su propio aprendizaje es así que se logrará que exista gusto por la lectura y sobre todo se den cuenta los estudiantes que leer les ayudará a superarse.

- Para lograr en los estudiantes interés por la lectura, se puede motivar con cuentos infantiles acordes a la edad, al grado de dificultades y que no sean tan extensos, no se debe centrarlos en el lenguaje o la literatura o leer por leerlos, además se deberá considerar que la clase social, el nivel cultural de los padres de familia y en ciertos casos la escasez de recursos didácticos hace que influyan directamente en la falta de 
interés por la lectura, es así que los docentes deberán agregar énfasis de motivación y creatividad al momento de compartir con los niños y niñas, de esta manera se logrará el desarrollo de la comprensión lectora.

\section{Referencias bibliográficas.}

Acurio Ponce, B., \& Nuñez Naranjo, A. (2019). Creo, juego y aprendo con estrategias y recursos para mejorar la comprensión lectora. 593 Digital Publisher CEIT, 4(2), 4459. doi:https://doi.org/10.33386/593dp.2019.2.87

Alonso, J., \& Mateos, M. (1985). Comprensión lectora: Modelos entrenamiento y evaluación. Infancia y Aprendizaje, 5-19. Obtenido de https://media.utp.edu.co/referenciasbibliograficas/uploads/referencias/articulo/27-comprension-lector-modelosentrenamiento-evolucion-infancia-y-aprendizajepdf-fnUv0-articulo.pdf

Anaya-Durand, A., \& Anaya-Huertas, C. (2010). ¿Motivar para aprobar o para aprender? Estrategias de motivación del aprendizaje para los estudiantes. Tecnológia, ciencia y educación, 5-14. Obtenido de https://www.redalyc.org/pdf/482/48215094002.pdf

Aquino, R. (2020). El Cuento: Estrategia Docente para la Comprensión Lectora. Obtenido de http://tesis.usat.edu.pe/bitstream/20.500.12423/2646/1/TL_AquinoZapataRosa.pdf

Asamblea Nacional Constituyente del Ecuador. (2008). Constitución de la República del Ecuador.

Asamblea Nacional de la República del Ecuador. (2010). LOES. En LEY ORGANICA DE EDUCACION SUPERIOR, LOES. Quito: Presidencia de la Republica del Ecuador.

Benavides, D. (13 de Mayo de 2013). Estrategias Didácticas para fomentar la Lectura Crítica . Obtenido de https://www.redalyc.org/pdf/551/55128038004.pdf

Bermúdez Jiménez, D., Casanova Ropero, K., \& Villarreal, E. A. (2015). Estrategias motivacionales dirigidas a estudiantes del ciclo básico, inscritos e la carrera de contaduría pública de la Facultad de Ciencias Económicas y Sociales de la Universidad de Carabobo.

Carranza, L. (2018). Factores que determinan la Falta de Motivación hacia la Lectura . Obtenido http://repository.ut.edu.co/bitstream/001/2466/1/T\%200945\%20601\%20CD6004\%2 0APROBADO\%20LUIYIS\%20ELIGIO\%20CARRANZA\%20RUIZ.pdf 
Carrasco Altamirano, A. (2003). La escuela puede enseñar estrategias de lectura y promover su regular empleo. Revista Mexicana de Investigación Educativa, 128-142. Obtenido de https://www.redalyc.org/pdf/140/14001708.pdf

Ferreri, E. (2015). Estrategias compensatorias en el proceso de lectura de una LE: un recorrido teórico hacia una implementación práctica. Universidad Tecnológica Nacional. Obtenido de https://fcf.unse.edu.ar/eventos/eici2015/contenido/pdf/02.pdf

Flores Guerrero, D. (2016). La importancia e impacto de la lectura, redacción y pensamiento crítico en la educación superior. Redalyc, 128-135. Obtenido de https://www.redalyc.org/pdf/853/85346806010.pdf

Hoyos Flórez, A. M., \& Gallego, T. M. (2017). Desarrollo de habilidades de comprensión lectora en niños y niñas de la básica primaria. Revista Virtual Universidad Católica del Norte(51), 23-45. Obtenido de https://www.redalyc.org/pdf/1942/194252398003.pdf

Jiménez Ortíz, M., \& Gordo Contreras, A. (2014). EL CUENTO INFANTIL: FACILITADOR DE PENSAMIENTO DESDE UNA EXPERIENCIA PEDAGÓGICA. 151-170. doi:ISSN 2216-0159

Jouini, K., \& Saud, K. (2005). ESTRATEGIAS INFERENCIALES EN LA COMPRENSIÓN LECTORA. Revista de educacion, 96-115. Obtenido de http://www.aldadis.net/revista4/04/imagen/04jouini.pdf

Kistemaker, M., \& Broeder, P. (2014). Fremdsprachenunterricht im Spannungsfeld zwischen Sprachwissen und Sprachkönnen. Saarbrücken: Thomas Tinnefeld. doi:https://www.academia.edu/10232366/School_language_and_the_role_of_multil ingualism_in_class

Marina, L. (2014). La Comprensión Lectora Desde El Contructivismo.

Marínez Chepe, A., Tocto, C., \& Palacios, L. (2015). La expresión oral en los niños y los cuentos. UCV HAcer, Revista de Investigación y Cultura, 116-120. doi:ISSN: 23058552

Mendoza Velazco, D. J. (2018). Estrategias didácticas para el fortalecimiento del proceso lector a nivel andragógico. UIDE, Universidad Internacional del Ecuador, 35-52. Obtenido de https://revistas.uide.edu.ec/index.php/innova/article/view/403

Ospina Rodríguez, J. (2006). La motivación, motor del aprendizaje. Revista Ciencias de la Salud, 158-160. Obtenido de https://www.redalyc.org/pdf/562/56209917.pdf 
Padial Ruz, R., \& Sáenz-López, p. (2013). Los cuentos populares/tradicionales en educación infantil. Una propuesta a través del juego. Revista de Educación, Motricidad e Investigación, 32-47. Obtenido de https://www.researchgate.net/profile/Rosario_Padial_Ruz/publication/331675411_L OS_CUENTOS_POPULARESTRADICIONALES_EN_EDUCACION_INFANTI L_UNA_PROPUESTA_A_TRAVES_DEL_JUEGO/links/5cbda076a6fdcc1d49a62 e58/LOS-CUENTOS-POPULARES-TRADICIONALES-EN-EDUCACION-

\section{INFANTI}

Parra Delgado, G. (2008). Cuento colectivo: El Castillo. Innovación y experiencias educativas, 1-13. Obtenido de https://archivos.csif.es/archivos/andalucia/ensenanza/revistas/csicsif/revista/pdf/Nu mero_12/GEMA_PARRA_1.pdf

Prieto, T. (17 de Enero de 2021). Actividades y estrategias de comprensión lectora en las aulas. Obtenido de http://www.jstor.org/stable/23766966

Ramírez Leyva, E. M. (2009). ¿Qué es leer? ¿Qué es la lectura?. Investigación bibliotecológica,. Scielo, 161-188. Obtenido de Recuperado en 15 de enero de 2021, de http://www.scielo.org.mx/scielo.php?script=sci_arttext\&pid=S0187358X2009000100007\&lng=es\&tlng=es.

Redondo Gonzalez, A. (2008). La comprensión lectora. Innovación y experiencias educativas, 1-8. doi:ISSN: 1988-6047

Rojas Camargo, G., \& Prieto Martínez, M. (2017). El cuento multimodal como strategia didáctica para el aprendizaje de la lectura. Educación y Ciencia, 297-312. Obtenido de https://revistas.uptc.edu.co/index.php/educacion_y_ciencia/article/view/10286

Serrabona, J. (2008). Los cuentos vivenciados: imaginación y movimiento. Facultat de Psicologia, Ciències de l'Educació i de L'Esport Blanquerna, 61-78. doi:ISSN 02138646

Thomson, N. (2012). Language Teaching Strategies and Techniques Used to Support Students Learning in a Language other than Their Mother Tounge. Kongsberg International School. Obtenido de https://ibo.org/contentassets/4ccc99665bc04f3686957ee197c13855/thompson_exec sum_8-29-12.pdf

Tirado, F., Santos, G., \& Tejero-Díez, D. (2013). La motivación como estrategia educativa: Un estudio en la enseñanza de la botánica. Scielo, 39(139), 79-92. Obtenido de http://www.scielo.org.mx/scielo.php?script=sci_arttext\&pid=S018526982013000100006\&lng=es\&tlng=es. 
ISSN: 2600-5859

Vol. 4, N¹.2, p. 119-130, marzo, 2021

Vega López, N. A., Bañales Faz, G., Reyna Valladares, A., \& Pérez Amaro, E. (2014). Enseñanza de estrategias para la comprensión de textos expositivos con alumnos de sexto grado de primaria. Scielo, 1047-1068. Obtenido de http://www.scielo.org.mx/scielo.php?script=sci_arttext\&pid=S1405$66662014000400003 \& \operatorname{lng}=e s \& t \operatorname{lng}=e s$.

Xhemajli, A. (2016). The role of the teacher in interactive teaching. International Journal of Cognitive Research in Science, Engineering and Education, 4(1), 31-38. doi:doi:10.5937/IJCRSEE1601031X

Yubero, S., \& Larrañaga, E. (2015). Lectura y universidad: hábitos lectores de los estudiantes universitarios de España y Portugal. Profesional De La Información, 24(6), 717-723. Obtenido https://revista.profesionaldelainformacion.com/index.php/EPI/article/view/epi.2015. nov.03

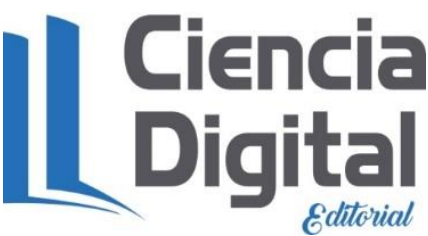




\section{PARA CITAR EL ARTÍCULO INDEXADO.}

Paredes Guevara, A. W., Núñez-Naranjo, A. F., Pérez, V., \& Yancha Yancha, K. R. (2021). El cuento: estrategia didáctica para la comprensión lectora . ConcienciaDigital, 4(1.2), 119130. https://doi.org/10.33262/concienciadigital.v4i1.2.1583

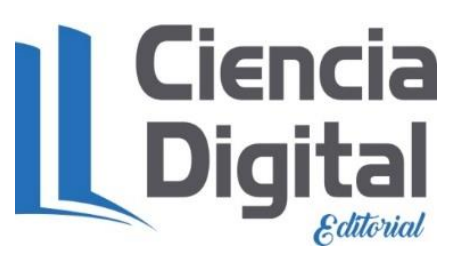

El artículo que se publica es de exclusiva responsabilidad de los autores y no necesariamente reflejan el pensamiento de la Revista Ciencia Digital.

El artículo queda en propiedad de la revista y, por tanto, su publicación parcial y/o total en otro medio tiene que ser autorizado por el director de la Revista Ciencia Digital.
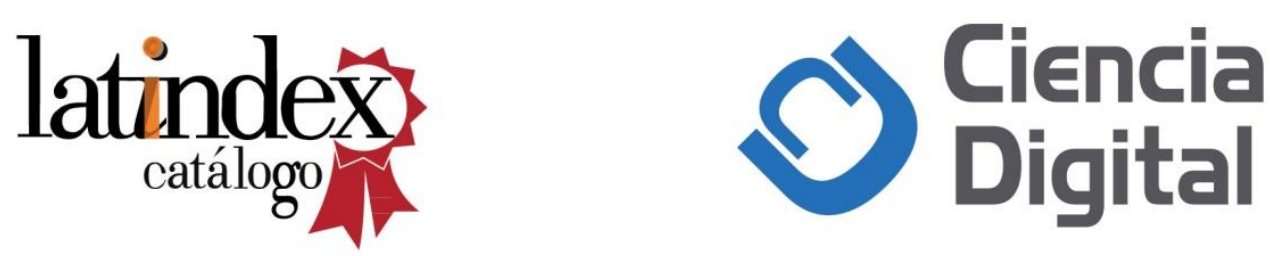